Supporting Information

\title{
A Synergistic Coreactant for Single-Cell Electrochemiluminescence Imaging: Guanine-Rich ssDNA-Loaded High-Index Faceted Gold Nanoflowers
}

\author{
Ying Chen, Xiaodan Gou, Cheng Ma,* Dechen Jiang* and Jun-Jie Zhu
}

State Key Laboratory of Analytical Chemistry for Life Science, School of Chemistry and Chemical Engineering, Nanjing University, Nanjing, Jiangsu, 210023, China

\section{Table of Content}

$\begin{array}{ll}\text { 1. Morphology and UV-Vis absorption spectra characterization. } & \text { S2 }\end{array}$

$\begin{array}{ll}\text { 2. ECL-potential curves and ECL enhancement factors. } & \text { S3 }\end{array}$

3. ECL spectrum and Electrochemical impedance spectroscopy. S4 


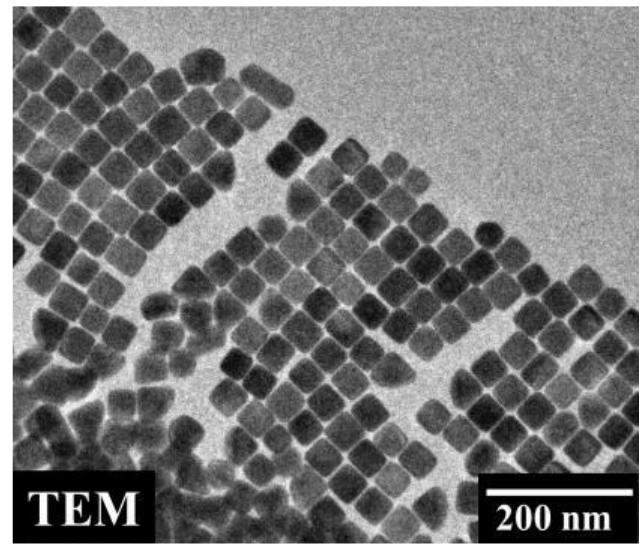

Figure S1. TEM image of Au nanocube seeds.

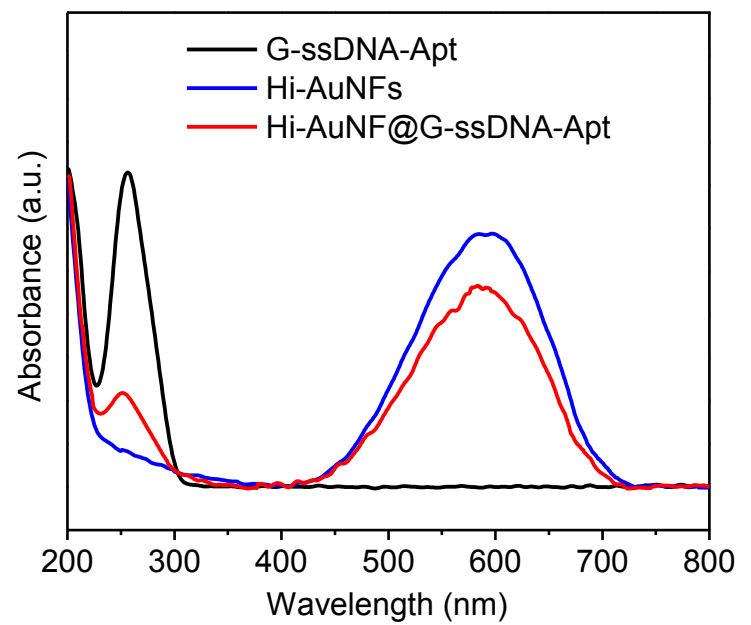

Figure S2. UV-Vis absorption spectra of G-ssDNA-Apt (black line), Hi-AuNFs (blue line) and HiAuNF@G-ssDNA-Apt nanocomposites (red line) solutions. 


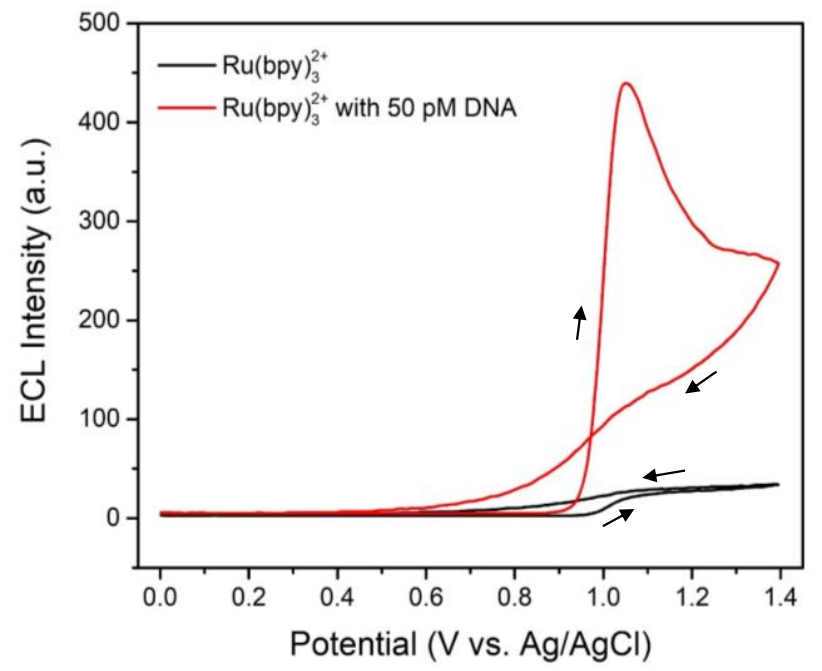

Figure S3. ECL-potential curves of $\mathrm{Ru}(\mathrm{bpy})_{3}{ }^{2+}$ in the absence of G-ssDNA and in the presence of 50 pM G-ssDNA. Work electrode is FTO.

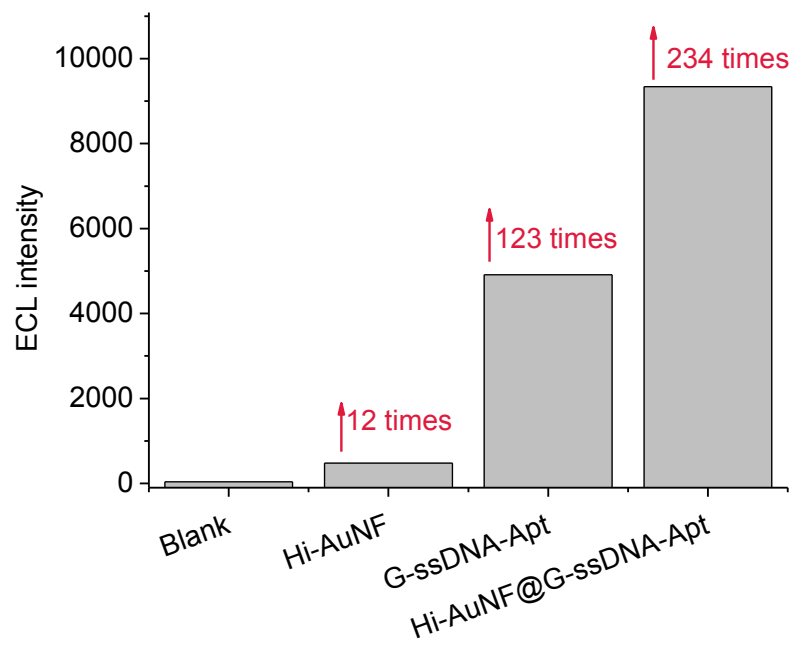

Figure S4. ECL enhancement factor of Hi-AuNF modified FTO, G-ssDNA-Apt modified FTO, and Hi-AuNF@G-ssDNA-Apt modified FTO, compared with bare FTO. The electrolyte is $200 \mu \mathrm{M}$ $\mathrm{Ru}($ bpy $) 3^{2+}$ (PBS buffer, $\mathrm{pH}$ 7.4). 


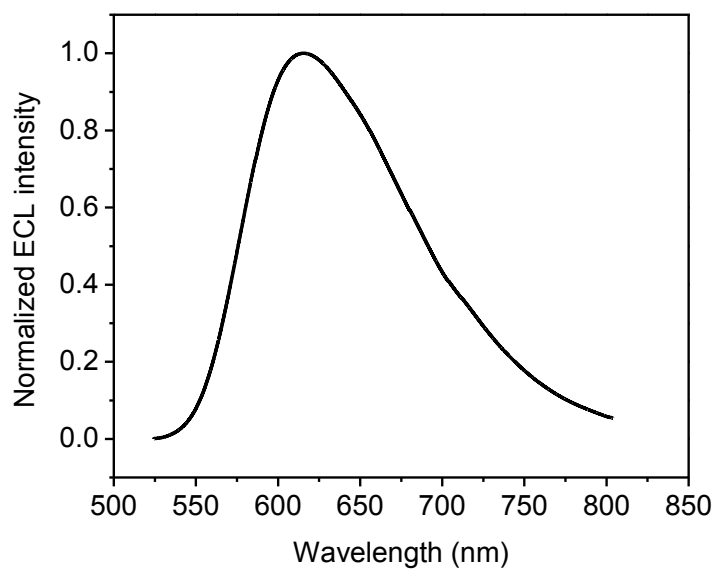

Figure S5. ECL spectrum of Ru(bpy) $3^{2+} / \mathrm{Hi}-\mathrm{AuNF} @ \mathrm{G}-\mathrm{ssDNA}$-Apt system.

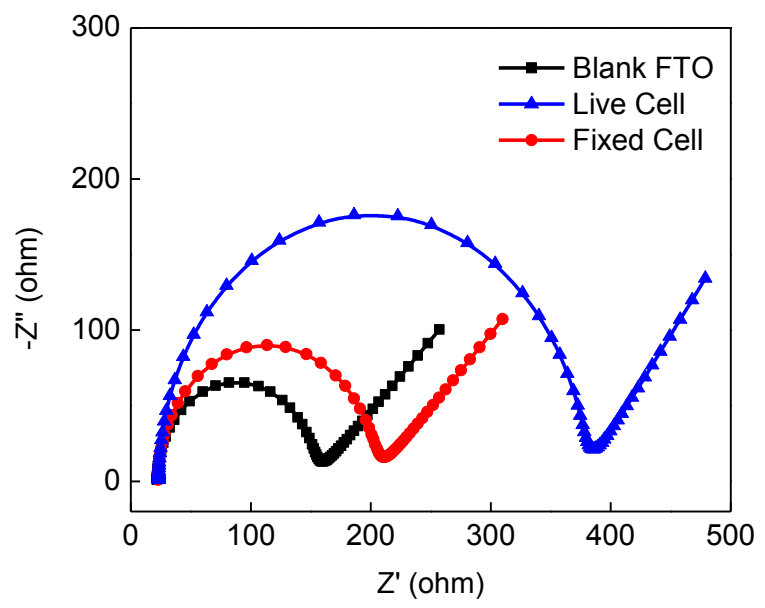

Figure S6. Electrochemical impedance spectroscopy of bare FTO (black line) and adherent living cells modified FTO before (blue line) and after (red line) cell fixation by paraformaldehyde and penetration by Triton X-100. 